\title{
Estrutura de rede quincunce em sistemas QAM-FBMC com filtros curtos
}

\author{
Iandra Galdino, Didier Le Ruyet, Marcello L. R. de Campos e Rostom Zakaria
}

Resumo - O sistema de banco de filtros com múltiplas portadoras baseado em símbolos QAM (QAM-FBMC) tem sido visado para suprir as necessidades dos sistemas de comunicação 5 G. Entretanto, para atingir a performance desejada é necessário o tratamento da interferência intrínseca do sistema. Com o objetivo de minimizar a interferência intrínseca, neste artigo nós avaliamos a aplicabilidade da estrutura de rede quincunce em sistemas QAM-FBMC, o qual utiliza filtros curtos. Nós comparamos e analisamos a performance do QAM-FBMC com estruturas de rede quincunce e retangular através da taxa de erro de bit (BER) incluindo os efeitos do canal AWGN e do canal EPA (Extended pedestrian A model).

Palavras-Chave-Banco de filtros, QAM-FBMC, 5G, estrutura de rede, rede quincunce.

Abstract-Quadrature Amplitude Modulation Filterbank Multicarrier (QAM-FBMC) has been a promising technology for $5 \mathrm{G}$ communication systems. However, to achieve the desired performance, it is necessary to deal with the intrinsic interference observed at the receiver. Aiming to overcome the intrinsic interference, in this paper, we evaluate the applicability of a quincunx lattice structure to the QAM-FBMC systems implemented with short filters. We compare and analyze the performance of the QAM-FBMC with rectangular and quincunx lattice structures through the bit error rate (BER) over AWGN and Extended pedestrian A model (EPA).

Keywords-Filter-bank, QAM-FBMC, 5G, lattice structure, quincunx lattice.

\section{INTRODUÇÃO}

A fim de atender às exigências do sistema $5 \mathrm{G}$, diversas soluções de formas de onda têm sido propostas, dentre elas, podemos citar o banco de filtros com múltiplas portadoras baseado em símbolos QAM, QAM-FBMC, (do Inglês, Quadrature amplitude modulation filter-bank multicarrier) [1]. Nesta proposta, o sistema QAM-FBMC é composto por um único filtro protótipo, o qual tem sido alvo de diversos estudos [2], [3], [4]. Em uma abordagem similar, o sistema QAMFBMC foi reformulado [5], desta vez empregando dois filtros protótipos. Para esta configuração com dois filtros novas propostas de filtro protótipo também têm sido estudados [6], [7] visando minimizar a interferência intrínseca do sistema.

Em sistemas com múltiplas portadoras, a duração do símbolo e o espaçamento entre as subportadoras define a estrutura de rede do sistema de comunicação [8]. Normalmente os

Iandra Galdino e Marcello L. R. de Campos, Universidade Federal do Rio de Janeiro UFRJ, Rio de Janeiro, Brasil, e-mails: iandra.galdino@smt.ufrj.br, campos@smt.ufrj.br. Rostom Zakaria e Didier Le Ruyet, Conservatoire national des arts et métiers CNAM, Paris 75141, França, e-mails: rostom.rostom22@gmail.com, leruyet@cnam.fr.

O presente trabalho foi realizado com apoio da Coordenação de Aperfeiçoamento de Pessoal de Nível Superior - Brasil (CAPES) - Código de Financiamento 001 sistemas são projetados em rede retangular, entretanto, os autores em [9] propuseram a utilização de sinais distribuídos com a mesma escala de tempo e frequência do canal, ou seja, utilizando estrutura de rede diferente da clássica retangular.

Diversas estruturas de rede foram estudadas em [10], inclusive a quincunce. A rede quincunce também é conhecida na literatura como rede hexagonal, uma vez que a interferência em torno de um ponto de interesse está distribuída nos vértices de um hexágono. Os autores em [11] propuseram a utilização da estrutura de rede quincunce em sistemas de comunicação. Eles apresentaram o sistema LOFDM, (do Inglês, Lattice $O F D M$ ), baseado na estrutura não retangular e demonstraram que o LOFDM supera o desempenho do tradicional OFDM em canais duplamente dispersivos. Apesar de encontrarmos na literatura o estudo de redes hexagonais em sistemas de comunicação, o sistema QAM-FBMC, quando utilizando filtros curtos, permanece sem esta avaliação, o que motivou o desenvolvimento deste trabalho.

Neste artigo avaliamos a aplicabilidade de estruturas de redes diferentes da tradicional (retangular) ao sistema QAMFBMC quando este utiliza filtros curtos, ou seja ovelapping factor $K=1$. Para isto, foram considerados sistemas QAMFBMC que utilizam apenas um filtro protótipo. Sabendo que a interferência intrínseca do sistema está relacionada à estrutura de rede utilizada, a interferência foi avaliada considerando diferentes redes, retangular e quincunce. Sabendo ainda que o filtro protótipo utilizado também influencia na interferência observada, e consequentemente no desempenho do sistema, diferentes filtros curtos foram aplicados ao sistema para avaliar a proposta.

Um projeto e otimização de filtros protótipo curtos para sistemas QAM-FBMC foi recentemente proposto em [2]. Nele os autores propõem minimizar a interferência enquanto mantêm a energia fora da banda de interesse, OOBE (do Inglês, Out-ofband energy) limitada. Sendo assim, neste trabalho avaliamos o desempenho do filtro OPF- $\beta$ (do Inglês, Optimized Prototype filter with $\mathrm{OOBE}=\beta$ ) proposto em [2] em sistemas QAMFBMC utilizando a estrutura de rede quincunce e retangular. Para efeitos de comparação, utilizamos ainda o filtro protótipo QMF (do Inglês, Quadrature mirror filter) [12], sendo que ambos possuem aproximadamente a mesma OOBE.

Uma vez que o objetivo deste trabalho é atender aos critérios do sistema 5G estabelecidos pela 3GPP, foram utilizados apenas filtros denominados curtos, cujo fator de sobreposição adotado (do Inglês, overlapping factor) foi $K=1$, visando a atender a demanda por baixa latência.

A relevância do trabalho apresentado consiste em verificar se a alteração da estrutura da rede utilizada contribui para 
a redução da interferência intrínseca do sistema. Consequentemente, reduzindo a interferência o desempenho do sistema poderá ser aprimorado.

Este trabalho é estruturado da seguinte maneira: o sistema QAM-FBMC com diferentes estruturas de rede é apresentado na Seção II. Na Seção III a interferência intrínseca é apresentada. Na Seção IV a estrutura de rede proposta é avaliada. Finalmente, as conclusões são apresentadas na Seção V.

\section{SistemA QAM-FBMC}

Diante da impossibilidade de utilização do sistema OQAMFBMC (do Inglês, Offset Quadrature Amplitude Modulation Filter-bank Multicarrier) em aplicações do tipo MIMO (do Inglês, multiple-input multiple-output) devido à interferência intrínseca do sistema observada [13], o sistema QAM-FBMC foi proposto em [1]. A ideia central do QAM-FBMC é modificar o sistema OQAM-FBMC convencional de forma que os símbolos QAM transmitidos não sejam mais deslocados no tempo, e assim fazer com que a interferência intrínseca possa ser reduzida.

Nesta seção nós apresentamos um modelo para sistema QAM-FBMC quando utilizando a estrutura de rede retangular (tradicional), conforme apresentado na Figura 1(a), e também um modelo do sistema QAM-FBMC usando a estrutura de rede quincunce, como representado na Figura 1(b).

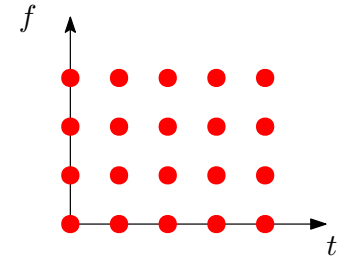

(a)

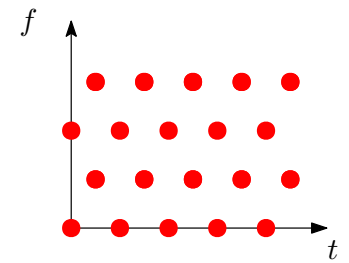

(b)
Fig. 1. Estrutura de rede: (a) Retangular, (b) Quincunce.

A estrutura de rede está relacionada ao espaçamento entre sub-portadoras e ao espaçamento entre símbolos. Ela pode ser definida por um conjunto de coordenadas dos filtros de um banco de filtros no plano tempo-frequência [11], [14]. $\mathrm{Na}$ prática ela pode ser gerada amostrando o plano tempofrequência nos pontos, conforme exemplificado na Figura 1.

A geometria da estrutura de rede pode ser descrita pela sua matriz geradora $L$ [15]. A matriz geradora contem em suas colunas as coordenadas dos primeiros símbolos da rede. As demais coordenadas são obtidas pela multiplicação da matriz $L$ pelo vetor $\left[n^{\prime} k^{\prime}\right]^{T}$, sendo que $k^{\prime}$ representa a $k^{\prime}$-ésima subportadora enviadas no $n^{\prime}$-ésimo símbolo QAM-FBMC. Por exemplo, a matriz

$$
L_{R}=\left[\begin{array}{cc}
T & 0 \\
0 & F
\end{array}\right]
$$

define uma estrutura retangular $\Lambda_{R}$, como aquela da Figura 1 (a), com duração de símbolo $T$ e espaçamento entre subportadoras $F$. Já a matriz geradora

$$
L_{Q}=\left[\begin{array}{cc}
T & 0.5 T \\
0 & F
\end{array}\right]
$$

define uma estrutura quincunce $\Lambda_{Q}$ [11], [14], como aquela exemplificada na Figura 1 (b).

\section{A. QAM-FBMC com estrutura de rede retangular}

Neste trabalho consideramos o sistema QAM-FBMC com um único filtro protótipo [1]. Desta forma, a expressão do sinal transmitido $s(t)$ em tempo contínuo considerando a estrutura de rede retangular pode ser descrita como:

$$
s(t)=\sum_{n^{\prime}} \sum_{k^{\prime}=0}^{M-1} d_{k^{\prime}, n^{\prime}} g\left(t-n^{\prime} T\right) e^{j 2 \pi F k^{\prime} t},
$$

onde $d_{k^{\prime}, n^{\prime}}$ são símbolos QAM complexos alocados na $k^{\prime}$ ésima sub-portadora e enviados no $n^{\prime}$-ésimo símbolo QAMFBMC. A duração do símbolo é representada por $T$, e o espaçamento entre subportadoras por $F$. O filtro protótipo utilizado $g(t)$ tem comprimento definido por $L=K M$, sendo $M$ a quantidade de sub-portadoras do sistema e $K$ um número inteiro que indica o fator de sobreposição do filtro (do inglês, overlapping factor). Neste trabalho o fator de sobreposição adotado foi $K=1$, o que ocasiona em filtros que denominamos curtos (em comparação aos demais $K>1$ ), visando à baixa latência necessária para sistema $5 \mathrm{G}$.

No receptor, considerando o canal do tipo ruidoso AWGN (do Inglês, Aditive white Gaussian noise), o $n$-ésimo símbolo demodulado pelo $k$-ésimo filtro casado pode ser definido por

$$
\tilde{d}_{k, n}=d_{k, n}+\underbrace{\sum_{\left(k^{\prime}, n^{\prime}\right) \neq(k, n)} d_{k^{\prime}, n^{\prime}} A_{g}\left(\Delta_{n} T, \Delta_{k} F\right)}_{\text {interferência }}+v_{k, n},
$$

sendo $v_{k, n}$ a representação do ruído, e $A_{g}\left(\Delta_{n} T, \Delta_{k} F\right)$ a função de ambiguidade [16], $\Delta_{n}=n-n^{\prime}$, e $\Delta_{k}=k-k^{\prime}$.

Sabendo que os filtros de transmissão e recepção não são perfeitamente ortogonais, podemos observar na recepção a presença da interferência intrínseca do sistema.

\section{B. QAM-FBMC com estrutura de rede quincunce}

Seja um sistema QAM-FBMC em banda base implementado com estrutura de rede quincunce conforme exemplificado na Figura 1, e com $M$ sub-portadoras. Considerando $d_{k^{\prime}, n^{\prime}}$ os símbolos transmitidos alocados na $k^{\prime}$-ésima subportadora e $n^{\prime}$-ésimo símbolo QAM-FBMC, o sinal transmitido pode ser descrito como [17]

$$
s(t)=\sum_{n^{\prime}} \sum_{k^{\prime}=0}^{M-1} d_{k^{\prime}, n^{\prime}} g\left(t-n^{\prime} T-\frac{\bmod \left(k^{\prime}, 2\right)}{2} T\right) e^{j 2 \pi F k^{\prime} t},
$$

sendo que $\bmod (a, b)$ representa o resto da divisão de $a$ por $b$.

Mais uma vez considerando o canal ruidoso AWGN, no receptor o $n$-ésimo símbolo demodulados na $k$-ésima subportadora pode ser descrito como:

$$
\begin{aligned}
& \tilde{d}_{k, n}=d_{k, n}+v_{k, n} \\
& +\underbrace{\sum_{\substack{\left(k^{\prime}, n^{\prime}\right) \\
\neq \\
(k, n)}} d_{k^{\prime}, n^{\prime}} A_{g}\left(\Delta_{n}^{\prime} T, \Delta_{k} F\right) e^{j 2 \pi\left(k-k^{\prime}\right)\left(\frac{\bmod (k, 2)-\bmod \left(k^{\prime}, 2\right)}{2}-n^{\prime}\right)}}_{\text {interferência }}
\end{aligned}
$$


sendo $\Delta_{n}^{\prime}=(n+1 / 2 \bmod (k, 2))-\left(n^{\prime}+1 / 2 \bmod \left(k^{\prime}, 2\right)\right)$.

Como podemos notar, similar ao que acontece quando utilizamos a estrutura de rede retangular, no caso quincunce também observamos a interferência intrínseca do sistema.

\section{INTERFERÊNCIA INTRÍNSECA}

A interferência intrínseca do sistema observada na subportadora $k$ na recepção vem dos símbolos vizinhos $\left(k^{\prime}, n^{\prime}\right)$, em razão do filtro protótipo empregado, e pode variar com a estrutura de rede utilizada. Sabendo que a interferência intrínseca afeta diretamente o desempenho do sistema, a escolha do filtro protótipo e da estrutura de rede utilizados tornam-se de fundamental importância para seu bom funcionamento.

A interferência total observada na subportadora de recepção $k$ de um sistema QAM-FBMC com estrutura de rede retangular pode ser obtida utilizando da Eq. (4) como

$$
I_{k, n}=\sum_{\left(k^{\prime}, n^{\prime}\right) \in \Upsilon_{k, n}} d_{k^{\prime}, n^{\prime}} \Gamma_{\Delta_{n}, \Delta_{k}}
$$

sendo $\Gamma_{\Delta_{n}, \Delta_{k}}=A_{g}\left(\Delta_{n} T, \Delta_{k} F\right)$ os coeficientes de interferência obtidos pela amostragem da função de ambiguidade do filtro protótipo, e $\Upsilon_{k, n}$ o conjunto de $\left(k^{\prime}, n^{\prime}\right)$ para o qual coeficiente de interferência não é nulo. Para exemplificar o processo, na Figura 2 nós apresentamos a função de ambiguidade do filtro protótipo QMF [12]. As marcações em vermelho representam os pontos $\left(\Delta_{n} T, \Delta_{k} F\right)$ em que devemos amostrar a função de ambiguidade para obter os coeficientes de interferência no caso da rede retangular, exceto pelo ponto central $(0,0)$, onde a informação é transmitida.

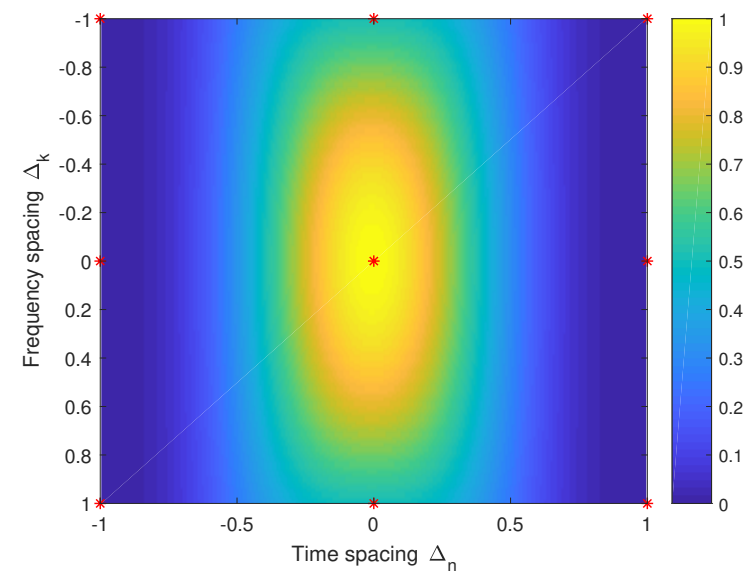

Fig. 2. Função de ambiguidade do filtro protótipo QMF, $\left(\left|A_{g}(T, F)\right|\right)$ com estrutura de rede retangular.

Considerando o sistema QAM-FBMC com estrutura de rede quincunce, a interferência total pode ser obtida através da Eq. (6) como

$$
I_{k, n}^{\prime}=\sum_{\left(k^{\prime}, n^{\prime}\right) \in \Upsilon_{k, n}} d_{k^{\prime}, n^{\prime}} \Gamma_{\Delta_{n}^{\prime}, \Delta_{k}}
$$

sendo $\Gamma_{\Delta_{n}^{\prime}, \Delta_{k}}=A_{g}\left(\Delta_{n}^{\prime} T, \Delta_{k} F\right) e^{-j 2 \pi \Delta_{k}\left(n^{\prime}-\frac{1}{2} \bmod \left(k^{\prime}, 2\right)\right)}$ os coeficientes de interferência do caso quincunce.

Para exemplificar, nós apresentamos na Figura 3 a função de ambiguidade do filtro QMF com marcações em vermelho

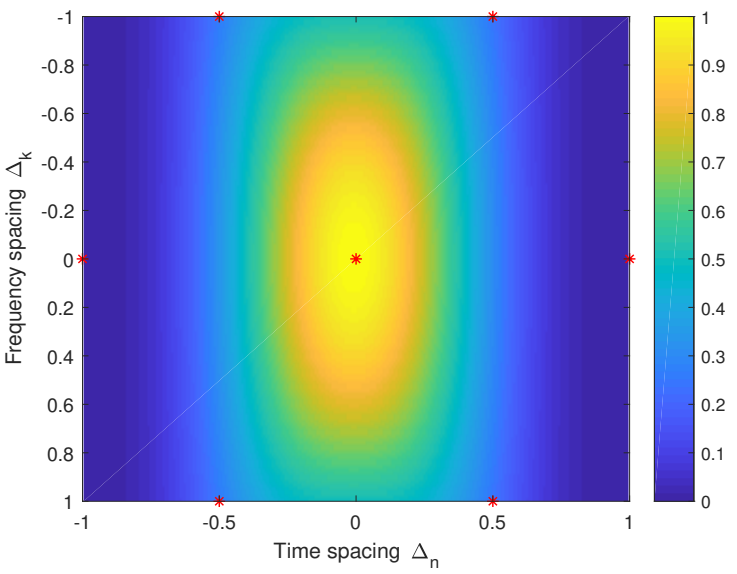

Fig. 3. Função de ambiguidade do filtro protótipo QMF, $\left(\left|A_{g}(T, F)\right|\right)$ com estrutura de rede quincunce.

$\left(\Delta_{n}^{\prime} T, \Delta_{k} F\right)$, as quais indicam onde devemos amostrar para obter os coeficientes de interferência.

Como podemos observar, amostrando a função de ambiguidade podemos obter o que denominamos tabela de interferência, ou seja, uma tabela com os coeficientes de interferência específicos de cada filtro e de cada estrutura de rede.

A Tabela I apresenta os coeficientes de interferência observados quando usamos estrutura de rede retangular e o filtro protótipo QMF.

TABELA I

COEF. DE INTERFERÊNCIA: $\Gamma_{\Delta_{n}, \Delta_{k}}$ REDE RETANGULAR E FILTRO QMF.

\begin{tabular}{c|ccc}
\hline$\Delta_{k}$ & \multicolumn{3}{|c}{$\Delta_{n}$} \\
& -1 & 0 & +1 \\
\hline-1 & 0 & $-0,5$ & 0 \\
0 & 0 & 0 & 0 \\
+1 & 0 & $-0,5$ & 0 \\
\hline
\end{tabular}

Nós apresentamos ainda na Tabela II os coeficientes obtidos quando utilizamos o filtro protótipo OPF- $\beta$ e a rede retangular.

TABELA II

COEF. DE INTERFERÊNCIA: $\Gamma_{\Delta_{n}, \Delta_{k}}$ REDE RETANGULAR E FILTRO OPF- $\beta$

\begin{tabular}{c|ccc}
\hline$\Delta_{k}$ & \multicolumn{3}{|c}{$\Delta_{n}$} \\
& -1 & 0 & +1 \\
\hline-2 & 0 & $0,0059-0,0003 \mathrm{i}$ & 0 \\
-1 & 0 & $-0,4646+0,0114 \mathrm{i}$ & 0 \\
0 & 0 & 0 & 0 \\
+1 & 0 & $-0,4646-0,0114 \mathrm{i}$ & 0 \\
+2 & 0 & $0,0059+0,0003 \mathrm{i}$ & 0 \\
\hline
\end{tabular}

Para comparação, apresentamos também na Tabela III e Tabela IV os coeficientes de interferência quando utilizamos a estrutura de rede quincunce e os filtros QMF e OPF- $\beta$ respectivamente. Como podemos observar, os coeficientes de interferência variam significativamente de acordo com o filtro protótipo e com a estrutura de rede utilizada.

Da análise das tabelas, podemos obter a interferência total do sistema relacionada ao filtro protótipo e ao tipo de estrutura de rede utilizados. Conforme apresentado na Tabela V, o sistema com estrutura quincunce apresenta significativa redução da interferência total. 
TABELA III

COEF. DE INTERFERÊNCIA: $\Gamma_{\Delta_{n}^{\prime}, \Delta_{k}}$ REDE QUINCUNCE E FILTRO QMF.

\begin{tabular}{c|ccc}
\hline$\Delta_{k}$ & & $\Delta_{n}^{\prime}$ \\
& $-0,5$ & 0 & 0,5 \\
\hline-1 & $-0,25 \mathrm{i}$ & $+0,25 \mathrm{i}$ & 0 \\
0 & 0 & 0 & 0 \\
+1 & $+0,25 \mathrm{i}$ & $-0,25 \mathrm{i}$ & 0 \\
\hline
\end{tabular}

TABELA IV

COEF. DE INTERFERÊNCIA: $\Gamma_{\Delta_{n}^{\prime}, \Delta_{k}}$ REDE QUINCUNCE E FILTRO OPF- $\beta$.

\begin{tabular}{c|ccc}
\hline$\Delta_{k}$ & - & $\Delta_{n}^{\prime}$ & \\
& $-0,5$ & 0 & $+0,5$ \\
\hline-5 & $-0,0024-0,0192 \mathrm{i}$ & $0,0024+0,0192 \mathrm{i}$ & 0 \\
-4 & 0 & $-0,0024+0,0002 \mathrm{i}$ & 0 \\
-3 & $-0,0020-0,0277 \mathrm{i}$ & $0,0020+0,0277 \mathrm{i}$ & 0 \\
-2 & 0 & $0,0059-0,0003 \mathrm{i}$ & 0 \\
-1 & $-0,0064-0,2627 \mathrm{i}$ & $0,0064+0,2627 \mathrm{i}$ & 0 \\
0 & 0 & 0 & 0 \\
+1 & $-0,0064+0,2627 \mathrm{i}$ & $0,0064-0,2627 \mathrm{i}$ & 0 \\
+2 & 0 & $0,0059+0,0003 \mathrm{i}$ & 0 \\
+3 & $-0,0020+0,0277 \mathrm{i}$ & $0,0020-0,0277 \mathrm{i}$ & 0 \\
+4 & 0 & $-0.0024-0,0002 \mathrm{i}$ & 0 \\
+5 & $-0,0024+0,0192 \mathrm{i}$ & $0,0024-0,0192 \mathrm{i}$ & 0 \\
\hline
\end{tabular}

TABELA V

INTERFERÊNCIA TOTAL.

\begin{tabular}{|c|c|c|}
\hline Filtro & retangular $[\mathrm{dB}]$ & quincunce $[\mathrm{dB}]$ \\
\hline \hline QMF & $-3,0103$ & $-6,0206$ \\
\hline OPF- $\beta$ & $-3,6452$ & $-5,4641$ \\
\hline
\end{tabular}

\section{Resultados DE SimulaÇÃo}

A aplicabilidade da estrutura de rede quincunce no sistema QAM-FBMC foi avaliada considerando filtros protótipo curtos $(K=1)$ QMF (do Inglês, Quadrature mirror filter) e OPF$\beta$, apresentado em [2] cujo comprimento foi definido sendo $L=K M=128$. Ambos os filtros utilizados possuem a mesma energia fora da banda de interesse, OOBE (do Inglês, Out-of-band energy). Ademais, o comprimento do símbolo foi definido como $M=128$ subportadoras. Com esta finalidade, as taxas de erro de bit do sistema, BER (do Inglês, Bit error rate), foram avaliadas sob a perspectiva do canal AWGN e do canal EPA (Extended pedestrian A model) e comparadas às taxas de erro do sistema QAM-FBMC com estrutura de rede tradicional, ou seja, retangular.

Na Figura 4 comparamos a performance do sistema QAMFBMC com estrutura de rede tradicional (retangular), modulação 4-QAM em canal ruidoso AWGN utilizando os filtros OPF- $\beta$ e QMF. Conforme esperado, pela análise da interferência apresentada na Tabela V, a performance do filtro OPF- $\beta$ é significativamente melhor do que a do QMF quando utilizamos a rede retangular, mesmo aplicando o cancelamento iterativo de interferência IIC (do Inglês, Iterative interference cancelation). No caso do filtro QMF, apresentamos apenas a curva QMF+IIC1, uma vez que não houve alteração significativa com mais iterações.

Nós comparamos ainda o desempenho do sistema QAMFBMC usando a estrutura de rede retangular e a estrutura quincunce, ambas com o filtro $\mathrm{OPF}-\beta$, quando consideramos o canal EPA, conforme apresentado na Figura 5.

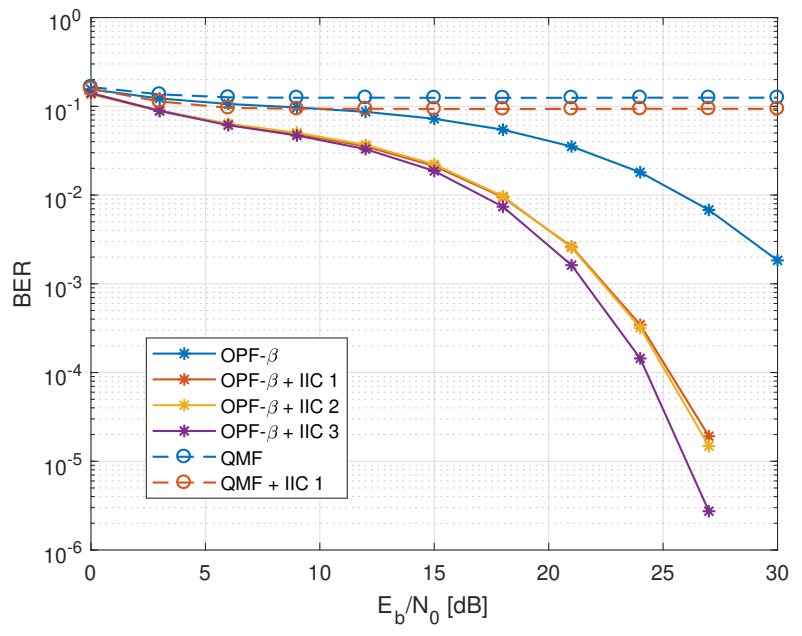

Fig. 4. Performance do sistema QAM-FBMC com estrutura de rede retangular, modulação 4-QAM, filtros OPF- $\beta$ e QMF, e canal AWGN.

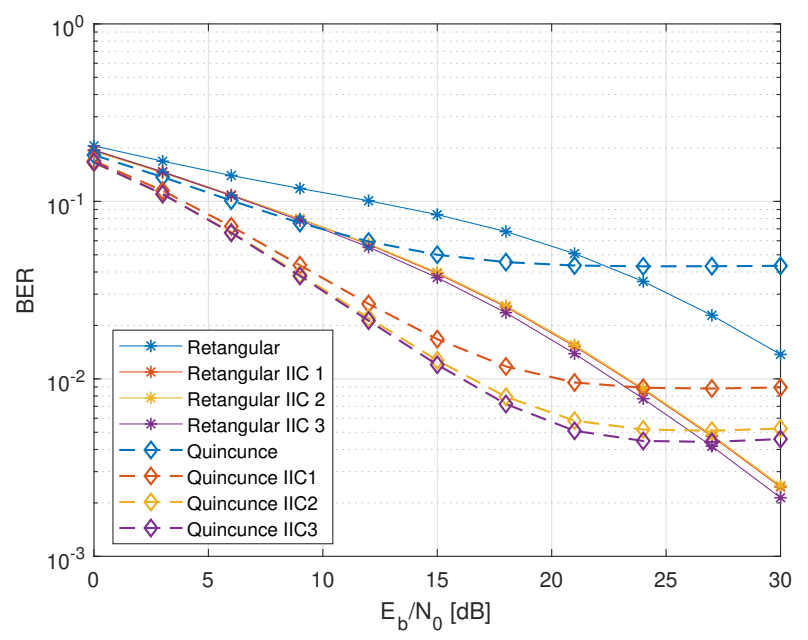

Fig. 5. Performance do sistema QAM-FBMC utilizando estrutura de rede retangular e quincunce, modulação 4-QAM, filtro protótipo OPF- $\beta$ e canal EPA.

Alterando a estrutura de rede do sistema QAM-FBMC de retangular para quincunce e utilizando o filtro OPF- $\beta$, o desempenho do sistema melhora significativamente para a relação $E_{b} / N_{0}$ inferior a aproximadamente $21 \mathrm{~dB}$. Essa melhora pode ser notada inclusive quando aplicamos o cancelamento iterativo de interferência, IIC. Entretanto, aumentando a relação $E_{b} / N_{0}$, a rede retangular apresenta melhor desempenho. Isso se deve a forma como os coeficientes de interferência estão distribuídos na tabela de interferência.

Ainda, para avaliar o benefício do uso da estrutura de rede quincunce em termos de BER, nós comparamos o desempenho do sistema QAM-FBMC usando a estrutura de rede retangular e quincunce quando o sistema emprega o filtro QMF sob o canal EPA, conforme apresentado na Figura 6.

Quando utilizamos o filtro protótipo QMF, o desempenho do sistema QAM-FBMC utilizando a estrutura de rede quincunce é significativamente melhor do que quando utilizando a estrutura retangular. Ainda, quando aplicamos 3 iterações de cancelamento de interferência (IIC), o desempenho do sistema 


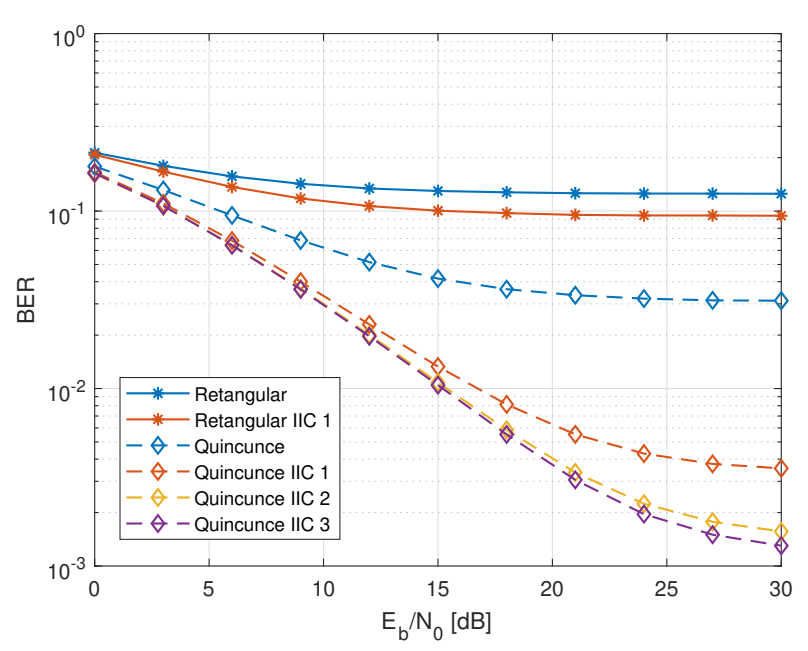

Fig. 6. Performance do sistema QAM-FBMC utilizando estrutura de rede retangular e quincunce, modulação 4-QAM, filtro protótipo QMF e canal EPA.

com rede quincunce apresenta significativo ganho.

Finalmente foram comparados o desempenho do sistema QAM-FBMC com rede retangular e com rede quincunce quando utilizando ambos os filtros protótipo, QMF e OPF- $\beta$ conforme apresentado na Figura 7.

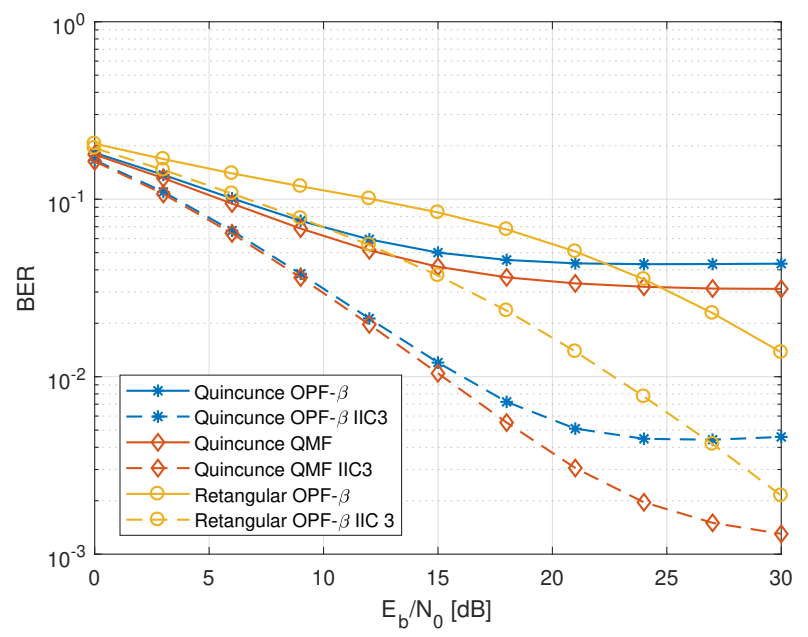

Fig. 7. Performance do sistema QAM-FBMC utilizando estrutura de rede retangular e quincunce, modulação 4-QAM, filtro protótipo QMF e OPF- $\beta$ e canal EPA.

Comparando o desempenho do sistema QAM-FBMC após 3 iterações IIC, a melhor performance é observada quando o sistema QAM-FBMC utiliza estrutura de rede quincunce e o filtro protótipo QMF.

\section{CONCLUSÕES}

A interferência observada em sistemas QAM-FBMC está diretamente relacionada ao filtro protótipo utilizado. Sua maior incidência pode ser constatada quando se trata de filtros curtos $(K=1)$, cuja capacidade de confinamento espectral é notoriamente inferior àquela de filtros longos i.e. $K \geq 1$.

Foi avaliada neste artigo a aplicabilidade da estrutura de rede quincunce no sistema QAM-FBMC com filtros protótipo curtos $(K=1)$ encontrados na literatura.
Nós mostramos que a estrutura de rede quincunce pode ser utilizada em sistemas QAM-FBMC com filtros curtos de forma a reduzir a interferência intrínseca do sistema. Desta forma, foram empregados diferentes filtros para avaliar o sistema em termos de taxa de erro de bit (BER) sob a perspectiva do canal AWGN e também do canal EPA.

$\mathrm{O}$ trabalho apresentado constatou um significativo ganho em termos da interferência observada pelo uso da rede quincunce. Nós constatamos ainda, com os resultados obtidos por simulação, que o desempenho do sistema QAM-FBMC pode ser significativamente melhorado com o uso da rede quincunce associado ao cancelamento iterativo de interferência quando utilizamos o filtro QMF.

\section{REFERÊNCIAS}

[1] R. Zakaria and D. Le Ruyet, "Intrinsic interference reduction in a filter bank-based multicarrier using QAM modulation," Physical Communication, vol. 11, pp. 15-24, 2014.

[2] I. Galdino, R. Zakaria, D. Le Ruyet, and M. L. R. de Campos, "Short-Filter design for intrinsic interference reduction in QAM-FBMC modulation," IEEE Communications Letters, vol. 24, pp. 1487-1491, 2020.

[3] S. Taheri, M. Ghoraishi, P. Xiao, L. Zhang, and Y. Xin, "SquareRoot Nyquist Filter Design for QAM-Based Filter Bank Multicarrier Systems," IEEE Transactions on Vehicular Technology, vol. 67, no. 9, pp. 9006-9010, 2018.

[4] H. Kim, H. Han, and H. Park, "Waveform design for QAM-FBMC systems," in 18th International Workshop on Signal Processing Advances in Wireless Communications (SPAWC). IEEE, 2017, pp. 1-5.

[5] H. Nam, M. Choi et al., "A New Filter-Bank Multicarrier System With Two Prototype Filters for QAM Symbols Transmission and Reception," IEEE Transactions on Wireless Communications, vol. 15, no. 9, pp. 5998-6009, 2016.

[6] Y. Qi and M. Al-Imari, "An enabling waveform for 5G QAM-FBMC: Initial analysis," in Conference on Standards for Communications and Networking (CSCN). IEEE, 2016, pp. 1-6.

[7] Y. H. Yun, C. Kim et al., "A new waveform enabling enhanced QAMFBMC systems," in 16th International Workshop on Signal Processing Advances in Wireless Communications (SPAWC). IEEE, 2015, pp. 116120.

[8] A. Sahin, I. Guvenc, and H. Arslan, "A survey on multicarrier communications: Prototype filters, lattice structures, and implementation aspects," IEEE communications surveys \& tutorials, vol. 16, no. 3, pp. 1312-1338, 2013.

[9] B. Le Floch, M. Alard, and C. Berrou, "Coded orthogonal frequency division multiplex [tv broadcasting]," Proceedings of the IEEE, vol. 83 no. 6, pp. 982-996, 1995.

[10] J. H. Conway and N. J. A. Sloane, Sphere packings, lattices and groups. Springer Science \& Business Media, 2013, vol. 290.

[11] T. Strohmer and S. Beaver, "Optimal OFDM design for time-frequency dispersive channels," IEEE Transactions on communications, vol. 51, no. 7, pp. 1111-1122, 2003.

[12] H. S. Malvar, "Modulated QMF filter banks with perfect reconstruction," Electronics Letters, vol. 26, no. 13, pp. 906-907, 1990.

[13] R. Zakaria, D. Le Ruyet, and Y. Medjahdi, "On isi cancellation in mimo$\mathrm{ml}$ detection using fbmc-qam modulation," in International Symposium on Wireless Communication System (ISWCS), 2012, pp. 1-5.

[14] J. H. Conway and N. J. A. Sloane, Sphere packings, lattices and groups. Springer-Verlag New York, 1993.

[15] A. Sahin, I. Guvenc, and H. Arslan, "A Survey on Multicarrier Communications: Prototype Filters, lattice Structures, and Implementation Aspects," IEEE Communications Surveys \& Tutorials, vol. 16, no. 3, pp. 1312-1338, 2014.

[16] P. M. Woodward, Probability and Information Theory, with Applications to Radar. Pergamon London, 1953.

[17] S. Peng, A. Liu, X. Pan, and H. Wang, "Hexagonal multicarrier fasterthan-Nyquist signaling," IEEE Access, vol. 5, pp. 3332-3339, 2017. 